\title{
Jejunal Metabolic Responses to Escherichia coli Infection in Piglets
}

\author{
Hucong Wu't, Jiaqi Liu't, Siyuan Chen², Yuanyuan Zhao', Sijing Zeng'², Peng Bin², \\ Dong Zhang ${ }^{1}$, Zhiyi Tang ${ }^{2 *}$ and Guoqiang Zhu ${ }^{1 *}$

\begin{abstract}
${ }^{1}$ College of Veterinary Medicine, Jiangsu Co-Innovation Center for Important Animal Infectious Diseases and Zoonoses, University, Yangzhou, China, ${ }^{2}$ Guangdong Provincial Key Laboratory of Animal Nutrition Control, Institute of Subtropical Animal Nutrition and Feed, College of Animal Science, South China Agricultural University, Guangzhou, China
\end{abstract} \\ Joint International Research Laboratory of Agriculture and Agri-Product Safety of Ministry of Education of China, Yangzhou
}

OPEN ACCESS

Edited by:

Jie Yin,

Institute of Subtropical

Agriculture (CAS), China

Reviewed by:

Luchang Zhu,

Houston Methodist Research

Institute, United States

Yongjie Liu,

Nanjing Agricultural University, China

Charles Z. Li,

Agricultural Research Service (USDA),

United States

*Correspondence:

Zhiyi Tang

43591680@qq.com

Guoqiang Zhu

yzgqzhu@yzu.edu.cn

tThese authors have contributed equally to this work

Specialty section:

This article was submitted to

Food Microbiology,

a section of the journal

Frontiers in Microbiology

Received: 12 July 2018

Accepted: 26 September 2018

Published: 16 October 2018

Citation:

Wu H, Liu J, Chen S, Zhao Y, Zeng S,

Bin $P$, Zhang $D$, Tang $Z$ and Zhu G

(2018) Jejunal Metabolic Responses

to Escherichia coli Infection in Piglets.

Front. Microbiol. 9:2465.

doi: 10.3389/fmicb.2018.02465
This study aimed to investigate the jejunal metabolic variations in enterotoxigenic Escherichia coli (ETEC)-infected piglets. Piglets were infected with $1 \times 10^{10}$ CFUs (colony-forming units) of ETEC W25K and assigned into diarrheal, recovered, control, and resistant groups. Jejunal samples were harvested at day 6 and metabolic profiles were analyzed via gas chromatography coupled to time-of-flight mass spectrometry (GC/TOFMS). The results showed that 33 metabolites in the jejunum were identified in ETEC-induced diarrhea, including amino acids, fatty acids, sugars, and organic acids. Compared with the control, resistant, and recovered piglets, diarrheal piglets showed higher concentrations of 4-aminobutyric acid (GABA) and glycine in the jejunum. Compared with the control and resistant piglets, six metabolites were markedly decreased in diarrheal piglets, including ornithine, asparagine, glutamine, citric acid, citrulline, and lysine. Collectively, this study provides insights into jejunal metabolic response to ETEC infection and ETEC induced diarrhea in piglets.

Keywords: jejunum, metabolism, ETEC, diarrhea, piglet

\section{INTRODUCTION}

Diarrheal illnesses are a severe public health problem and pathogenic enterotoxigenic Escherichia coli (ETEC) has been considered as a major cause of diarrhea in human and animals (Fleckenstein et al., 2010). After infection, ETEC rapidly colonizes in small intestine, including duodenum, jejunum, and ileum. ETEC colonization inhibits intestinal immune function and induces inflammatory response. In our previous report, we found that ETEC infection inhibits the mRNA expression of intestinal immune factors, such as polymeric immunoglobulin receptor (pIgR), cryptdin-related sequence 1C (CRS1C), and Reg3 $\gamma$ in mice (Liu et al., 2017). Meanwhile, ETEC infection upregulates intestinal IL-17 and causes dysbiosis of intestinal microbiota via increasing abundance of $\gamma$-aminobutyric acid (GABA)-producing Lactococcus lactis subsp. lactis (Ren et al., 2016b). The jejunal metabolite (e.g., amino acids and polyamine) participate in many important physiological process, such as the regulation of gene expression, synthesis and secretion of hormones, oxidative defense, and so on $(\mathrm{Wu}, 2009)$. The proteome analysis from our previous study identifies 92 differentially expressed proteins in the jejunum after exposure to ETEC and large body of these proteins were involved in metabolic process, such as protein turnover, nutrients (i.e., nucleotide, amino acids, carbohydrate, lipid, and inorganic ion) transport and metabolism, coenzyme metabolism, energy production and conversion, and secondary metabolite biosynthesis (Ren et al., 2016a). Metabolomics is an emerging analytical technique to seek global profiles of 
A

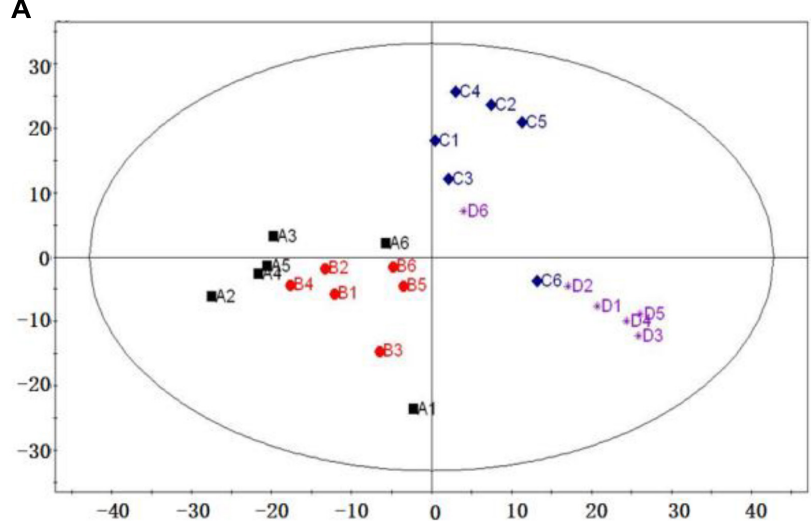

C

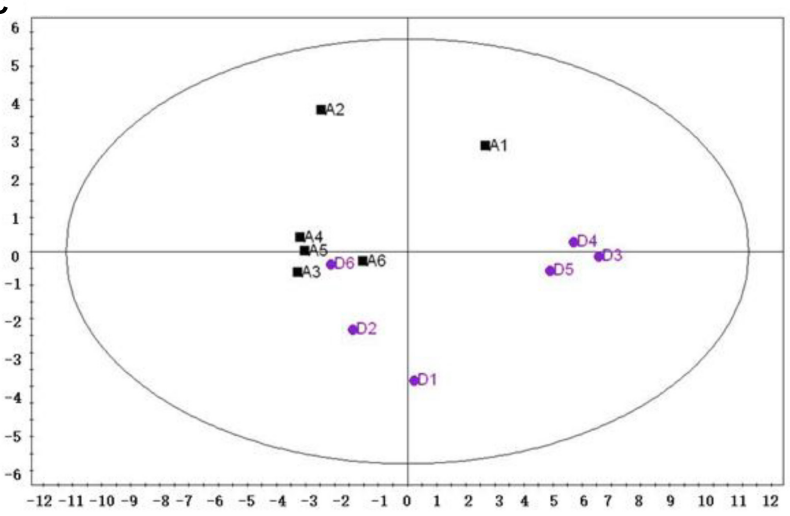

B

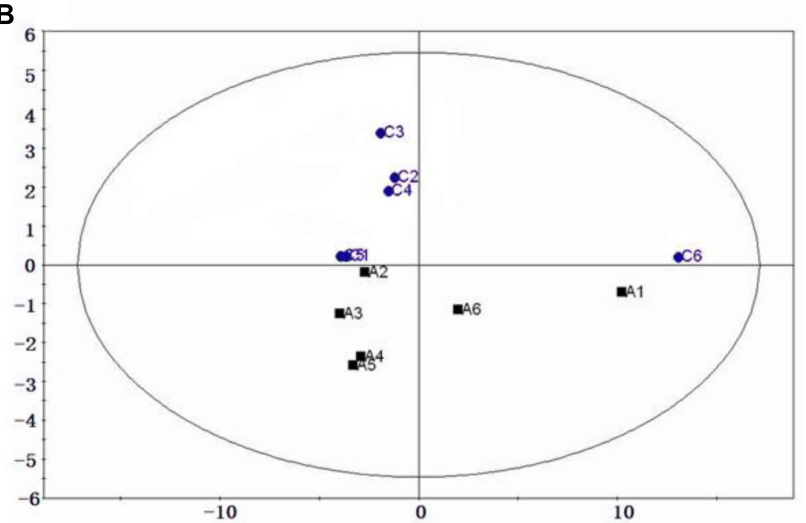

D

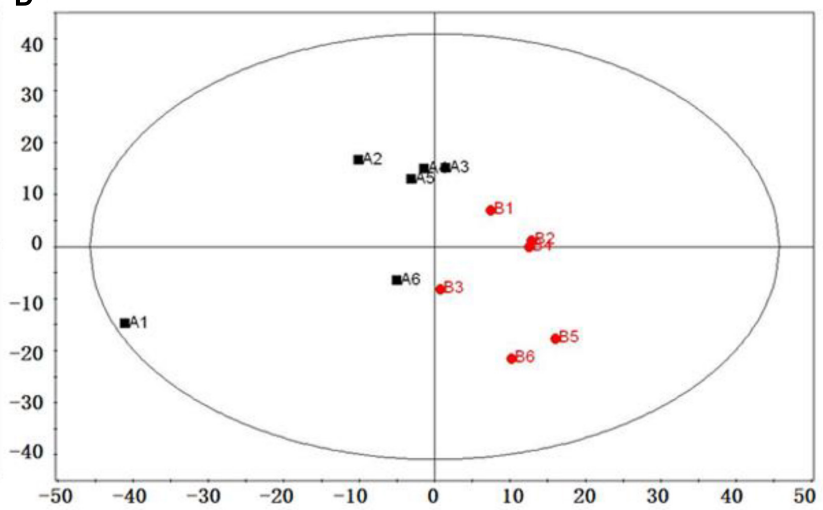

FIGURE 1 | Principal component analysis (PCA) score plot derived from the GC-MS analysis of jejunum from diarrheal piglets (black dots), recovered piglets (red dots), control piglets (blue dots), and resistant piglets (purple dots). (A) Total PCA, modeling diagnostic $R^{2} X=0.261 ; Q=0.0803$; (B) diarrheal piglets vs. control piglets, modeling diagnostic $R^{2} X=0.901 ; Q=0.295$; (C) diarrheal piglets vs. resistant piglets, modeling diagnostic $R^{2} X=0.734 ; Q=0.379$; and (D) diarrheal piglets vs. recovered piglets, modeling diagnostic $R^{2} X=0.286 ; Q=-0.163$.

metabolites in particular samples, including endogenous and exogenous metabolites (Khan et al., 2017). Therefore, we conduct this study to further investigate metabolic profiles in the jejunum after ETEC infection in piglets.

\section{MATERIALS AND METHODS}

\section{Bacterial Strains}

This study used the Escherichia coli F4-producing strain W25K (O149:K91, K88ac; LT, ST, EAST), which was originally isolated from a diarrheal piglet.

\section{ETEC Infection}

This study was conducted according to the guidelines of the Institute of Subtropical Agriculture, Chinese Academy of Sciences. Piglets (Landrace $\times$ Yorkshire; 18-day-old) were purchased from ZhengDa, Co., Chongqing, China and orally administrated with ETEC W25K at dose of $1 \times 10^{10}$ CFUs (colony-forming units) for five consecutive days (Ren et al., 2015, 2016a). The control piglets were treated with the same volume of LB medium. Fecal consistency was scored daily as: $0=$ normal;
$1=$ soft; 2 = runny or watery. Piglets with the development of watery diarrhea were defined as diarrheal piglets, and piglets that were recovered from diarrhea were regarded as recovery piglets, while piglets that were challenged with ETEC but not suffered from diarrhea were defined as resistant piglets. Six control piglets, six diarrheal piglets, six recovered piglets, and six resistant piglets were randomly selected for collecting the samples.

\section{Sample Preparation}

Twenty-four piglets were sacrificed at day 6 after ETEC infection and jejunal samples (100 mg) and extraction solvents $(50 \mu \mathrm{L} \mathrm{L}-$ 2-chlorophenylalanine and $350 \mu \mathrm{L}$ methanol) were added and then homogenized using a Mini-BeadBeater-16 (Biospec, Co., Bartlesville, OK, United States) for $5 \mathrm{~min}$. The mixture was placed on a shaker at $70^{\circ} \mathrm{C}$ for $10 \mathrm{~min}$ and centrifuged at $12,000 \times g$ and $4^{\circ} \mathrm{C}$ for $10 \mathrm{~min}$. The supernatant was separated, transferred into a $\mathrm{GC}$ vial, and then evaporated to dryness under a stream of $\mathrm{N}_{2}$ gas.

Methoxyamine hydrochloride $(20 \mu \mathrm{L}, 20 \mathrm{mg} / \mathrm{mL}$ pyridine) was added to the dried fraction and incubated at $37^{\circ} \mathrm{C}$ for $2 \mathrm{~h}$. One hundred $\mu \mathrm{L}$ of bis-(trimethylsilyl) trifluoroacetamide (BSTFA) containing 1\% TMCS was rapidly added and incubated 

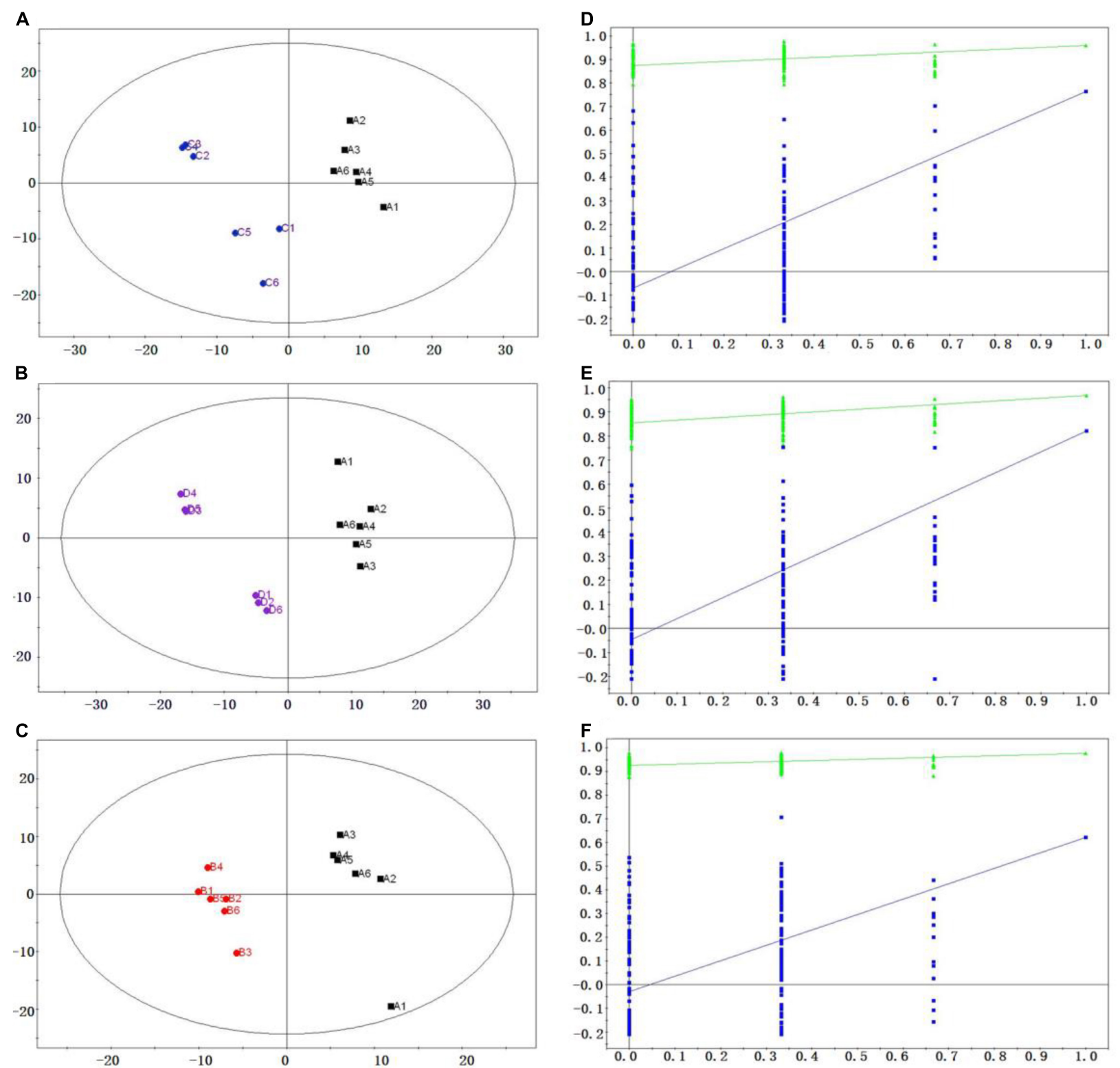

FIGURE 2 | Score plots and permutation test results for PLS-DA model derived from jejunum samples. Score plots of PLS-DA, (A) diarrheal piglets (black dots) vs. control piglets (blue dots); (B) diarrheal piglets (black dots) vs. resistant piglets (purple dots); (C) diarrheal piglets (black dots) vs. recovered piglets (red dots). Permutation validation of PLS-DA model, blue dots and green dots represented $\mathrm{Q}^{2}$ and $\mathrm{R}^{2}$, respectively; (D) diarrheal piglets vs. control piglets; (E) diarrheal piglets vs. resistant piglets; and (F) diarrheal piglets vs. recovered piglets.

at $70^{\circ} \mathrm{C}$ for $1 \mathrm{~h}$. Then the samples were kept at room temperature before analysis.

\section{GC-TOFMS Analysis}

Metabolites in jejunal samples were derivatized prior to gas chromatography coupled to time-of-flight mass spectrometry (GC-TOFMS) analysis (Agilent 7890A, Agilent, United States; LECO Chroma TOF PEGASUS 4D, MI, LECO, United States). The system utilized a DB-5MS capillary column coated with 5\% diphenyl cross-linked with 95\% dimethylpolysiloxane (30 $\mathrm{m} \times 250 \mu \mathrm{m}$ inner diameter, $0.25 \mu \mathrm{m}$ film thickness;
J\&W Scientific, Folsom, CA, United States). A $1 \mu \mathrm{L}$ aliquot of the analyte was injected in splitless mode. Helium was used as the carrier gas, the front inlet purge flow was $3 \mathrm{~mL} \min ^{-1}$, and the gas flow rate through the column was $1 \mathrm{~mL} \mathrm{~min}{ }^{-1}$. The initial temperature was kept at $90^{\circ} \mathrm{C}$ for $0.25 \mathrm{~min}$, then raised to $240^{\circ} \mathrm{C}$ at a rate of $5^{\circ} \mathrm{C} \mathrm{min}^{-1}$, and finally to $285^{\circ} \mathrm{C}$ at a rate of $20^{\circ} \mathrm{C} \mathrm{min}$ m $^{-1}$ for $11.5 \mathrm{~min}$. The injection, transfer line, and ion source temperatures were 280,250 and $220^{\circ} \mathrm{C}$, respectively. The energy was $-70 \mathrm{eV}$ in electron impact mode. The mass spectrometry data were acquired in full-scan mode with the $\mathrm{m} / \mathrm{z}$ range of $20-600$ 


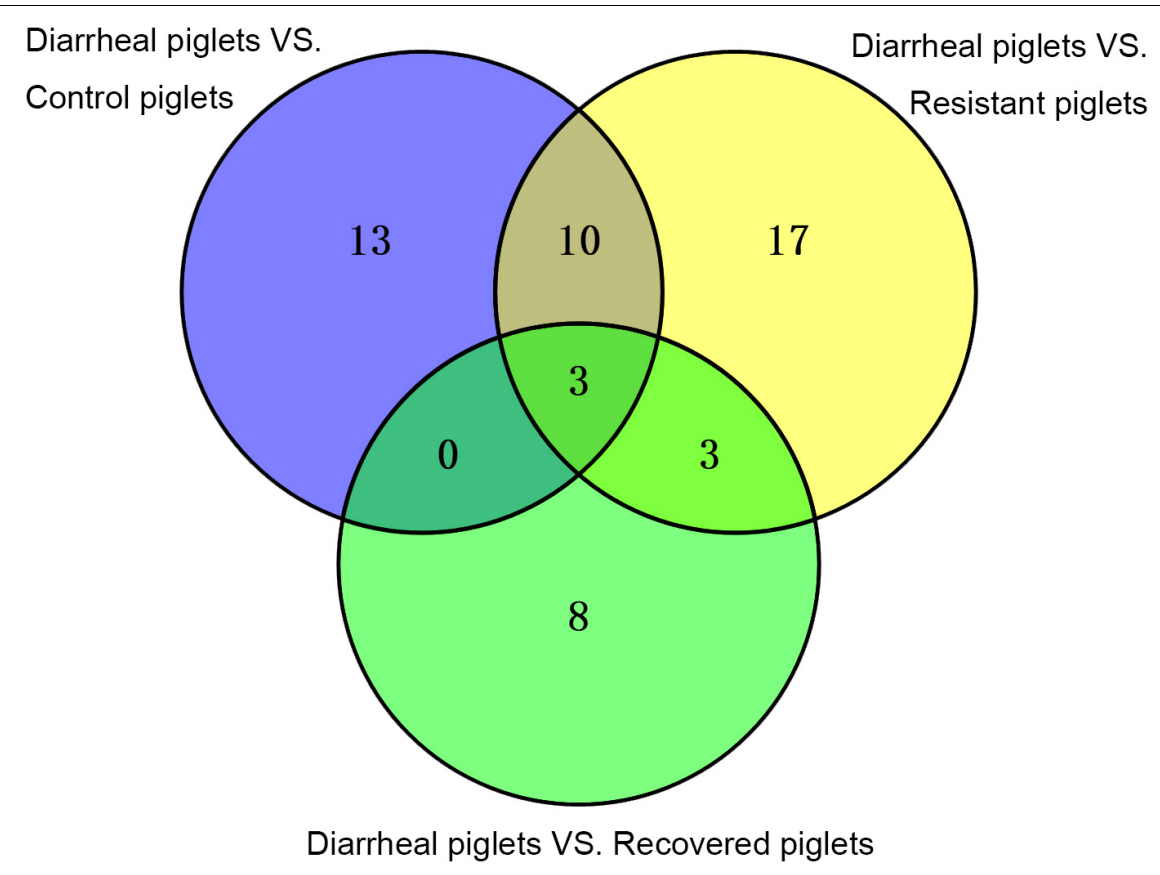

FIGURE 3 | Venn diagrams illustrating that similar differential metabolites (including increase and decrease) among diarrheal piglets vs. control piglets, diarrheal piglets vs. recovered piglets, and diarrheal piglets vs. resistant piglets.

at a rate of 100 spectra per second after a solvent delay of $492 \mathrm{~s}$.

\section{Data Processing and Analysis}

Each sample was represented by a GC-TOFMS chromatograph. The GC-TOFMS raw data were processed by Chroma TOF 4.3X software (LECO Corporation, St. Joseph, MI, United States) and LECO-Fiehn Rtx5 database for raw peaks extracting, data baselines filtering and calibration, peak alignment, deconvolution analysis, peak identification, and peak area integration. All the output data exported from Chroma TOF 4.3X software were imported into SIMCA-P software (version 11.0, Umetrics, Umeå, Sweden) for multivariate statistical analyses including a principal component analysis (PCA), partial least squares-discriminant analysis (PLS-DA), and pairwise orthogonal projections to latent structures discriminant analyses (OPLS-DA).

\section{RESULTS}

\section{PCA Model Analysis}

Principal component analysis is an unsupervised mathematical procedure used to identify latent structures in the dataset and outliers (Caboni et al., 2014). PCA of jejunal samples from diarrheal piglets, recovered piglets, control piglets, and resistant piglets was shown in Figure 1A. The results showed that plots from diarrheal piglets, recovered piglets, control piglets, and resistant piglets were separated each other. As the points that were close to each other had similar metabolic profiles, our results indicated that there might be significant metabolic differences among the four groups. The modeling of the three datasets (diarrheal piglets vs. control piglets, diarrheal piglets vs. resistant piglets, and diarrheal piglets vs. recovered piglets) of separate pairs, revealed separation between subjects (Figures 1B-D).

\section{PLS-DA Model Analysis}

To specify the metabolic variations produced by ETEC infection, PLS-DA models were constructed in jejunal samples (Figure 2). The results showed that the samples from each group were perfectly separated in three subjects: diarrheal piglets vs. control piglets $\left(\mathrm{R}^{2} \mathrm{X}=0.90, \mathrm{Q}^{2}=0.30\right)$, diarrheal piglets vs. resistant piglets $\left(\mathrm{R}^{2} \mathrm{X}=0.73, \mathrm{Q}^{2}=0.38\right)$ and diarrheal piglets vs. recovered piglets $\left(\mathrm{R}^{2} \mathrm{X}=0.29, \mathrm{Q}^{2}=-0.16\right)$. This phenomenon indicated that the physiological metabolism was interrupted by ETEC infection. In addition, diarrheal piglets showed distinctive metabolic profiles compared with piglets that recovered from diarrhea and were resistant to ETEC infection.

\section{OPLS-DA Model Analysis}

The variable importance in the projection (VIP) statistic of the first principal component of orthogonal partial least squares discriminant analysis (OPLS-DA) model (threshold $>1$ ) coupled with the $P$-value of the Student's $t$-test (threshold $<0.05$ ) were used for selecting significant variables responsible for group separation.

As shown in Figures 3, 4, the OPLS-DA models showed a clear separation between the diarrheal piglets vs. control piglets $\left(\mathrm{R}^{2} \mathrm{X}=0.41, \mathrm{R}^{2} \mathrm{Y}=0.96, \mathrm{Q}^{2}=0.77\right)$, diarrheal piglets vs. resistant piglets $\left(\mathrm{R}^{2} \mathrm{X}=0.45, \mathrm{R}^{2} \mathrm{Y}=0.97, \mathrm{Q}^{2}=0.82\right)$ and diarrheal 


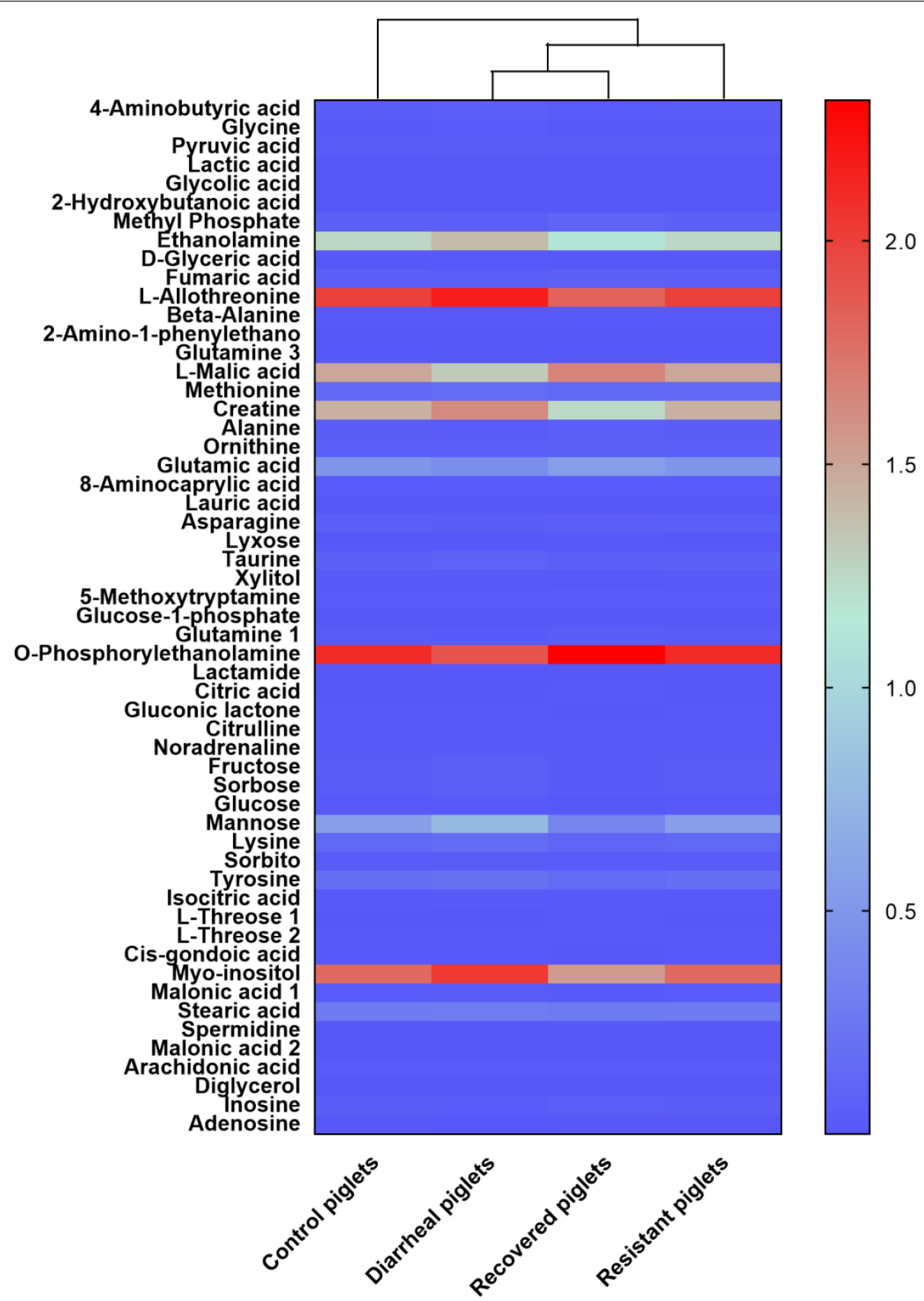

FIGURE 4 | The results from the analysis of differential metabolites were normalized by logarithmic and Euclidean distance and produced a heat map.

piglets vs. recovered $\left(\mathrm{R}^{2} \mathrm{X}=0.32, \mathrm{R}^{2} \mathrm{Y}=0.98, \mathrm{Q}^{2}=0.62\right)$. We detected 26, 33, and 14 differential metabolites between diarrheal piglets vs. control piglets, diarrheal piglets vs. resistant piglets and diarrheal piglets vs. recovered piglets, respectively. However, only three differential metabolites were commonly altered (including increase and decrease) among them.

Compared with the control piglets, diarrheal piglets showed higher concentration of nine metabolites in the jejunum [4-aminobutyric acid (GABA), glycine, 8-aminocaprylic acid, taurine, 5-methoxytryptamine, lactamide, isocitric acid, L-threose, and malonic acid]. However, 17 metabolites showed a decreased trend in the diarrheal piglets (2-hydroxybutanoic acid, L-allothreonine, 2-amino-1-phenylethanol, methionine, ornithine, lauric acid, asparagine, glutamine, $O$-phosphorylethanolamine, citric acid, citrulline, lysine, tyrosine, myo-inositol, stearic acid, spermidine and arachidonic acid) (Table $\mathbf{1}$ ).

Compared with the resistant piglets, nine metabolites were significantly enhanced in the diarrheal piglets (GABA, glycine, pyruvic acid, lactic acid, ethanolamine, creatine, 8-aminocaprylic 
TABLE 1 | The variation in content of metabolites in the jejunum between diarrheal and control piglets.

\begin{tabular}{|c|c|c|c|c|c|c|}
\hline Metabolites & RT & Cout & Mass & VIP & $P$-value & Fold change \\
\hline Lactamide & 16.08 & 12 & 257 & 1.81 & 0.002 & 271000.00 \\
\hline 4-Aminobutyric acid* & 5.53 & 17 & 69 & 1.92 & 0.001 & 4.33 \\
\hline 8-Aminocaprylic acid* & 13.60 & 24 & 174 & 1.89 & 0.001 & 3.10 \\
\hline 5-Methoxytryptamine & 15.27 & 24 & 174 & 1.42 & 0.032 & 2.71 \\
\hline L-Threose 2 & 19.48 & 24 & 73 & 1.44 & 0.029 & 2.28 \\
\hline Taurine & 14.20 & 24 & 326 & 1.58 & 0.013 & 2.25 \\
\hline Isocitric acid & 18.71 & 24 & 147 & 1.61 & 0.011 & 1.99 \\
\hline Glycine & 7.02 & 24 & 102 & 1.62 & 0.011 & 1.98 \\
\hline Malonic acid 1 & 20.77 & 23 & 122 & 1.42 & 0.031 & 1.67 \\
\hline 2-Amino-1-phenylethanol & 11.19 & 24 & 156 & 1.49 & 0.022 & 0.84 \\
\hline L-Allothreonine & 10.18 & 24 & 73 & 1.49 & 0.023 & 0.80 \\
\hline Methionine & 12.04 & 24 & 176 & 1.41 & 0.034 & 0.77 \\
\hline Tyrosine & 18.37 & 24 & 218 & 1.37 & 0.040 & 0.71 \\
\hline Ornithine & 13.31 & 24 & 142 & 1.52 & 0.018 & 0.68 \\
\hline Myo-inositol & 20.78 & 24 & 217 & 1.54 & 0.016 & 0.66 \\
\hline Citrulline & 16.51 & 24 & 157 & 1.70 & 0.006 & 0.64 \\
\hline Lysine & 18.09 & 24 & 156 & 1.51 & 0.019 & 0.64 \\
\hline Asparagine* & 14.12 & 24 & 116 & 1.93 & 0.001 & 0.56 \\
\hline Stearic acid & 22.74 & 24 & 117 & 1.56 & 0.015 & 0.54 \\
\hline Citric acid & 16.37 & 24 & 273 & 1.38 & 0.038 & 0.53 \\
\hline O-Phosphorylethanolamine & 15.87 & 24 & 73 & 1.67 & 0.008 & 0.51 \\
\hline 2-Hydroxybutanoic acid & 7.06 & 19 & 146 & 1.38 & 0.039 & 0.50 \\
\hline Arachidonic acid & 25.75 & 24 & 80 & 1.83 & 0.003 & 0.38 \\
\hline Glutamine 1 & 15.74 & 24 & 156 & 1.55 & 0.016 & 0.31 \\
\hline Lauric acid & 13.94 & 16 & 117 & 1.55 & 0.016 & 0.27 \\
\hline Spermidine & 22.83 & 20 & 174 & 1.41 & 0.033 & 0.25 \\
\hline
\end{tabular}

*The extremely significant metabolites between diarrheal and control piglets, which P-value $<0.001$.

acid, taurine, and noradrenaline), while 24 metabolites were significantly enhanced in the diarrheal piglets (beta-alanine, glutamine, L-malic acid, alanine, ornithine, glutamic acid, asparagine, lyxose, glucose-1-phosphate, citric acid, gluconic lactone, citrulline, fructose, sorbose, mannose, lysine, sorbitol, L-threose, spermidine, malonic acid, diglycerol, inosine, uridine monophosphate, and lactobionic acid) (Table 2).

Compared with the recovered piglets, 14 metabolites were significantly different in diarrheal piglets, and 8 metabolites were increased in the jejunum (GABA, glycine, glycolic acid, Dglyceric acid, xylitol, glucose, cis-gondoic acid, and malonic acid). Meanwhile, six metabolites were decreased in diarrheal piglets (methyl phosphate, fumaric acid, alanine, inosine, adenosine, and uridine monophosphate) (Table 3).

\section{DISCUSSION}

Infection with ETEC bacteria is the major cause of diarrhea in human and animals. After infection, ETEC rapidly colonizes the intestine and secretes exotoxins, which further disrupt intestinal barrier integrity and cause secretory diarrhea (Deng et al., 2015). In addition, ETEC colonization induces imbalance of intestinal microbiota and may dysregulate intestinal metabolism (Ren et al., 2016b). In this study, 33 metabolites have been identified in ETEC induced diarrhea, including amino acids, fatty acids, sugars, and organic acids.

Compared with the control, resistant and recovered piglets, diarrheal piglets have higher concentrations of GABA and glycine in the jejunum. GABA, a transmitter of enteric interneurons, has been noticed in the cytoplasm and the brush border of intestinal epithelial cells and regulates the function of the gastrointestinal tract (Wang et al., 2004; Li et al., 2012; Jung et al., 2017). The direct functions of intestinal GABAergic signaling system have been identified to be involved in fluid transport through luminal secretion of $\mathrm{Cl}^{-}$(Jin et al., 2006), which is a major driving force for fluid secretion and increased during diarrhea. Similar to our results in piglets, Li et al. (2012) reported that intestinal GABAergic signaling was upregulated in diarrheal mice caused by ovalbumin and blocking this GABA signaling decreased the occurrence of allergic diarrhea. In our previous study, we found that ETEC infection increased GABA-producing L. lactis subsp. lactis and GABA production, which further promotes IL-17 expression through mTORC1-S6K1-EGR-2-GFI-1 pathway and mediates intestinal inflammation (Ren et al., 2016b). Glycine serves as a precursor for glutathione along with cysteine and glutamic acid (Yin et al., 2016), while cysteine, glutamic acid and glutamine were markedly decreased in diarrheal piglets, suggesting that increased glycine failed to contribute to the glutathione synthesis and antioxidant effect in ETEC model. In intestinal injury, even after manifestation of a severe systemic 
TABLE 2 | The variation in content of metabolites in the jejunum between diarrheal and resistant piglets.

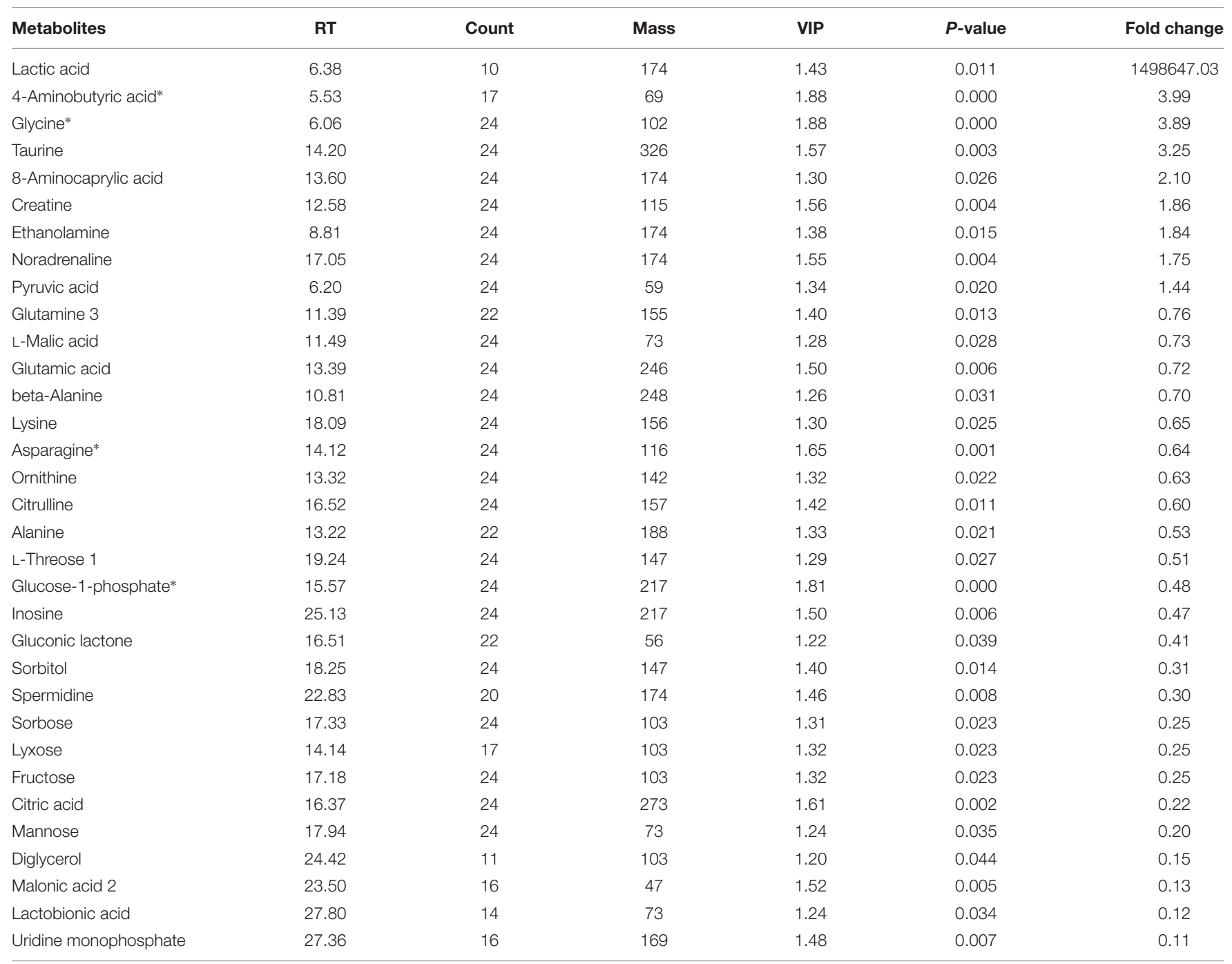

*The extremely significant metabolites between diarrheal and resistant piglets, which P-value $<0.001$.

TABLE 3 | The variation in content of metabolites in the jejunum between diarrheal and recovered piglets.

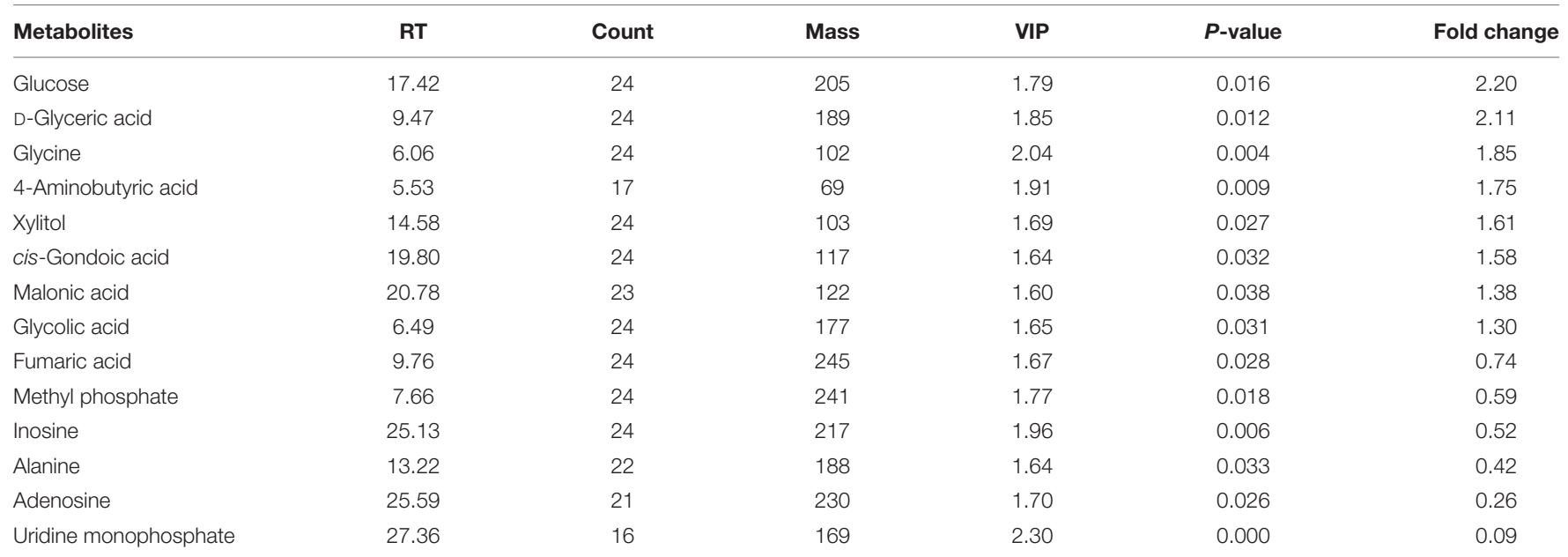


impairment (Effenberger-Neidnicht et al., 2014) and causing $\mathrm{pH}$ dependent membrane damage to ETEC (Vanhauteghem et al., 2012), glycine has been demonstrated to protect intestine against subacute endotoxemia. Meanwhile, the glycine cleavage system contributes to the intracellular replication of virulent bacterium and pathogenesis (Brown et al., 2014). Thus, the increased GABA and glycine in the jejunum may mediate or promote diarrhea in ETEC infectious piglet model.

Compared with the control and resistant piglets, we found that six metabolites were markedly decreased in diarrheal piglets, including ornithine, asparagine, glutamine, citric acid, citrulline, and lysine. Ornithine, asparagine, and citrulline play roles in the urea cycle (Mikulski et al., 2015), thus decreased ornithine and asparagine may indicate urea cycle is altered in ETEC induced diarrhea. Aspartate, a precursor of asparagine, has been demonstrated to enhance intestinal integrity and energy status in weaning piglets after lipopolysaccharide challenge ( $\mathrm{Pi}$ et al., 2014) and alleviate diquat-induced intestinal oxidative stress (Yin et al., 2015). Thus, the decreased jejunal asparagine in diarrheal piglets may exacerbate ETEC infection. Glutamine has been indicated to modulate intestinal permeability and tight junction expression in various diseases, and serves as a protective mechanism against radiation-induced diarrhea and diarrheapredominant irritable bowel syndrome (Kucuktulu et al., 2013; Bertrand et al., 2015). Lower jejunal glutamine in diarrheal piglets suggested that ETEC infection influenced glutamine synthesis, which may further disturbs the protective mechanism of glutamine against diarrhea. Citrate is an intermediate in the tricarboxylic acid cycle (TCA) and the reduced citrate in the diarrheal piglets suggested that the TCA was altered after ETEC infection.

Out of our expectation, we found that the lactic acid in diarrheal piglets was much higher than resistant piglets as a 1498647 fold change. Lactic acid was the metabolite fermented by various intestinal microorganisms (e.g., Klebsiella, Bacteroides, Lactobacillus), and presented in the intestine lumen (Zhao et al., 2011). As mentioned above, ETEC infection increased the relative abundance of lactic acid-producing bacteria (L. lactis subsp. lactis) significantly, thus the concentration of lactic acid in jejunum lumen was increased. But it failed to explain why lactic acid in jejunum tissue of diarrheal piglets was much higher than resistant piglets. In the healthy condition, the intestine

\section{REFERENCES}

Bertrand, J., Ghouzali, I., Guerin, C., Bole-Feysot, C., Gouteux, M., Dechelotte, P., et al. (2015). Glutamine restores tight junction protein Claudin-1 expression in colonic mucosa of patients with diarrhea-predominant irritable bowel syndrome. JPEN J. Parenter. Enteral Nutr. 40, 1170-1176. doi: 10.1177/ 0148607115587330

Brown, M. J., Russo, B. C., O'dee, D. M., Schmitt, D. M., and Nau, G. J. (2014). The contribution of the glycine cleavage system to the pathogenesis of Francisella tularensis. Microbes Infect. 16, 300-309. doi: 10.1016/j.micinf.2013.12.003

Caboni, P., Meloni, A., Lussu, M., Carta, E., Barberini, L., Noto, A., et al. (2014). Urinary metabolomics of pregnant women at term: a combined GC/MS and NMR approach. J. Mater. Fetal Med. 27, 4-12. doi: 10.3109/14767058.2014. 956403 of mammals was incapable of absorbing lactic acid, while the intestinal permeability might be changed in some pathological conditions, and the lactic acid was permitted to permeate the intestinal mucosa, thus the lactic acid may be regarded as an important indictor response to the intestinal barrier function (Qiu et al., 2013; Ikuta et al., 2017). We concluded that ETEC infection might impair the intestinal barrier of piglets and increase the permeability of jejunum (Yang et al., 2014), thus resulted in the high concentration of lactic acid in jejunum tissue.

\section{ETHICS STATEMENT}

All experimental protocols were approved by the Animal Care and Use Committee of Yangzhou University [approval ID: SYXK (Su) 2005-0005]. All animal care and use protocols in this study were performed in accordance with the approved current guidelines. At the end of the experimental period, all of the surviving or sick piglets were euthanized by potassium chloride.

\section{AUTHOR CONTRIBUTIONS}

GZ conceived and designed the study. HW, JL, SC, YZ, SZ, $\mathrm{PB}$, and $\mathrm{DZ}$ conducted experiments. HW and JL carried out animal experiments, performed statistical analysis, and drafted the manuscript. GZ finalized the manuscript. ZT conceived and designed the study and finalized the manuscript. All authors read and approved the final version of the manuscript.

\section{FUNDING}

This study was supported by National Basic Research Program of China (Grant No. 2013CB127301), National Natural Science Foundation of China (Grant Nos. 31272463 and 31472106), and Hunan Provincial Natural Science Foundation of China (Grant No. 12JJ2014). It was also funded by the Priority Academic Program of Development Jiangsu High Education Institution, and Innovation and Training Program of Jiangsu High Education Institution (201711117089X).

Deng, Y., Han, X., Tang, S., Xiao, W., Tan, Z., Zhou, C., et al. (2015). Magnolol and honokiol regulate the calcium-activated potassium channels signaling pathway in Enterotoxigenic Escherichia coli-induced diarrhea mice. Eur. J. Pharmacol. 755, 66-73. doi: 10.1016/j.ejphar.2015.03.002

Effenberger-Neidnicht, K., Jagers, J., Verhaegh, R., and De Groot, H. (2014). Glycine selectively reduces intestinal injury during endotoxemia. J. Surg. Res. 192, 592-598. doi: 10.1016/j.jss.2014.06.016

Fleckenstein, J. M., Hardwidge, P. R., Munson, G. P., Rasko, D. A., Sommerfelt, H., and Steinsland, H. (2010). Molecular mechanisms of enterotoxigenic Escherichia coli infection. Microbes Infect. 12, 89-98. doi: 10.1016/j.micinf.2009. 10.002

Ikuta, N., Chikamoto, K., Asano, Y., Yasui, Y., Yokokawa, H., Terao, K., et al. (2017). Time course effect of R-alpha-lipoic acid on cellular metabolomics in cultured hepatoma cells. J. Med. Food 20, 211-222. doi: 10.1089/jmf.2016.3837 
Jin, N., Kolliputi, N., Gou, D., Weng, T., and Liu, L. (2006). A novel function of ionotropic gamma-aminobutyric acid receptors involving alveolar fluid homeostasis. J. Biol. Chem. 281, 36012-36020. doi: 10.1074/jbc.M60689 5200

Jung, W. Y., Kim, S. G., Lee, J. S., Kim, H. K., Son, B. G., Kim, J. W., et al. (2017). Effect of feeding high gamma-aminobutyric acid-containing giant embryo black sticky rice (Oryza sativa L.) on Anxiety-Related Behavior of C57BL/6 Mice. J. Med. Food 20, 777-781. doi: 10.1089/jmf.2017.3928

Khan, A., Pan, J. H., Cho, S., Lee, S., Kim, Y. J., and Park, Y. H. (2017). Investigation of the hepatoprotective effect of Prunus mume Sieb. et Zucc extract in a mouse model of alcoholic liver injury through high-resolution metabolomics. J. Med. Food 20, 734-743. doi: 10.1089/jmf.2016.3874

Kucuktulu, E., Guner, A., Kahraman, I., Topbas, M., and Kucuktulu, U. (2013). The protective effects of glutamine on radiation-induced diarrhea. Support. Care Cancer 21, 1071-1075. doi: 10.1007/s00520-012-1627-0

Li, Y., Xiang, Y. Y., Lu, W. Y., Liu, C., and Li, J. (2012). A novel role of intestine epithelial GABAergic signaling in regulating intestinal fluid secretion. Am. J. Physiol. Gastrointest. Liver Physiol. 303, G453-G460. doi: 10.1152/ajpgi.00497. 2011

Liu, G., Ren, W., Fang, J., Hu, C. A., Guan, G., Al-Dhabi, N. A., et al. (2017). L-Glutamine and L-arginine protect against enterotoxigenic Escherichia coli infection via intestinal innate immunity in mice. Amino Acids 49, 1945-1954. doi: 10.1007/s00726-017-2410-9

Mikulski, T., Dabrowski, J., Hilgier, W., Ziemba, A., and Krzeminski, K. (2015). Effects of supplementation with branched chain amino acids and ornithine aspartate on plasma ammonia and central fatigue during exercise in healthy men. Folia Neuropathol. 53, 377-386. doi: 10.5114/fn.2015.56552

Pi, D., Liu, Y., Shi, H., Li, S., Odle, J., Lin, X., et al. (2014). Dietary supplementation of aspartate enhances intestinal integrity and energy status in weanling piglets after lipopolysaccharide challenge. J. Nutr. Biochem. 25, 456-462. doi: 10.1016/ j.jnutbio.2013.12.006

Qiu, J. W., Yan, M. Z., Wu, Y. N., Li, L. Z., and Tian, W. (2013). The effects of natural and modified clinoptilolite on intestinal barrier function and immune response to LPS in broiler chickens. Vet. Immunol. Immunopathol. 153, 70-76. doi: 10.1016/j.vetimm.2013.02.006

Ren, W., Yin, J., Chen, S., Duan, J., Liu, G., Li, T., et al. (2016a). Proteome analysis for the global proteins in the jejunum tissues of enterotoxigenic Escherichia coli -infected piglets. Sci. Rep. 6:25640. doi: 10.1038/srep25640

Ren, W., Yin, J., Xiao, H., Chen, S., Liu, G., Tan, B., et al. (2016b). Intestinal microbiota-derived GABA mediates interleukin-17 expression during enterotoxigenic Escherichia coli infection. Front. Immunol. 7:685. doi: 10.3389/ fimmu.2016.00685
Ren, W., Yin, J., Gao, W., Chen, S., Duan, J., Liu, G., et al. (2015). Metabolomics study of metabolic variations in enterotoxigenic Escherichia coli-infected piglets. RSC Adv. 5, 59550-59555. doi: 10.1039/C5RA0 9513A

Vanhauteghem, D., Janssens, G. P., Lauwaerts, A., Sys, S., Boyen, F., Kalmar, I. D., et al. (2012). Glycine and its $\mathrm{N}$-methylated analogues cause $\mathrm{pH}$-dependent membrane damage to enterotoxigenic Escherichia coli. Amino Acids 43, 245-253. doi: 10.1007/s00726-011-1068-y

Wang, F. Y., Watanabe, M., Zhu, R. M., and Maemura, K. (2004). Characteristic expression of gamma-aminobutyric acid and glutamate decarboxylase in rat jejunum and its relation to differentiation of epithelial cells. World J. Gastroenterol. 10, 3608-3611. doi: 10.3748/wjg.v10.i24. 3608

Wu, G. (2009). Amino acids: metabolism, functions, and nutrition. Amino Acids 37, 1-17. doi: 10.1007/s00726-009-0269-0

Yang, K. M., Jiang, Z. Y., Zheng, C. T., Wang, L., and Yang, X. F. (2014). Effect of Lactobacillus plantarum on diarrhea and intestinal barrier function of young piglets challenged with enterotoxigenic Escherichia coli K88. J. Anim. Sci. 92, 1496-1503. doi: 10.2527/jas.2013-6619

Yin, J., Liu, M., Ren, W., Duan, J., Yang, G., Zhao, Y., et al. (2015). Effects of dietary supplementation with glutamate and aspartate on diquat-induced oxidative stress in piglets. PLoS One 10:e0122893. doi: 10.1371/journal.pone. 0122893

Yin, J., Ren, W., Yang, G., Duan, J., Huang, X., Fang, R., et al. (2016). 1-Cysteine metabolism and its nutritional implications. Mol. Nutr. Food Res. 60, 134-146. doi: 10.1002/mnfr.201500031

Zhao, Y., Qin, G., Sun, Z., Che, D., Bao, N., and Zhang, X. (2011). Effects of soybean agglutinin on intestinal barrier permeability and tight junction protein expression in weaned piglets. Int. J. Mol. Sci. 12, 8502-8512. doi: 10.3390/ ijms 12128502

Conflict of Interest Statement: The authors declare that the research was conducted in the absence of any commercial or financial relationships that could be construed as a potential conflict of interest.

Copyright (C) $2018 \mathrm{Wu}$, Liu, Chen, Zhao, Zeng, Bin, Zhang, Tang and Zhu. This is an open-access article distributed under the terms of the Creative Commons Attribution License (CC BY). The use, distribution or reproduction in other forums is permitted, provided the original author(s) and the copyright owner(s) are credited and that the original publication in this journal is cited, in accordance with accepted academic practice. No use, distribution or reproduction is permitted which does not comply with these terms. 